\title{
Touchscreen-based location discrimination and paired associate learning tasks detect cognitive impairment at an early stage in an App knock-in mouse model of Alzheimer's disease
}

\author{
Md. Ali Bin Saifullah', Okiru Komine ${ }^{2}$, Yutao Dong ${ }^{1,3}$, Kazuya Fukumoto ${ }^{1}$, Akira Sobue ${ }^{2}$, Fumito Endo ${ }^{2}$, \\ Takashi Saito ${ }^{2,4,5}$, Takaomi C. Saido ${ }^{4}$, Koji Yamanaka ${ }^{2}$ and Hiroyuki Mizoguchi ${ }^{1,3^{*}}$ (D)
}

\begin{abstract}
Alzheimer's disease (AD) is a progressive neurodegenerative disorder characterized by cognitive decline with accumulation of amyloid beta $(A \beta)$ and neurofibrillary tangles that usually begins 15-30 years before clinical diagnosis. Rodent models that recapitulate aggressive $A \beta$ and/or the pathology of neurofibrillary tangles are essential for $A D$ research. Accordingly, non-invasive early detection systems in these animal models are required to evaluate the phenotypic changes, elucidate the mechanism of disease progression, and facilitate development of novel therapeutic approaches. Although many behavioral tests efficiently reveal cognitive impairments at the later stage of the disease in AD models, it has been challenging to detect such impairments at the early stage. To address this issue, we subjected 4-6-month-old male App ${ }^{N L-G-F / N L-G-F}$ knock-in (App-KI) mice to touchscreen-based location discrimination (LD), different object-location paired-associate learning (dPAL), and reversal learning tests, and compared the results with those of the classical Morris water maze test. These tests are mainly dependent on the brain regions prone to $A \beta$ accumulation at the earliest stages of the disease. At 4-6 months, considered to represent the early stage of disease when mice exhibit initial deposition of $A \beta$ and slight gliosis, the classical Morris water maze test revealed no difference between groups, whereas touchscreen-based LD and dPAL tasks revealed significant impairments in task performance. Our report is the first to confirm that a systematic touchscreen-based behavioral test battery can sensitively detect the early stage of cognitive decline in an AD-linked App-KI mouse model. This system could be applied in future translational research.
\end{abstract}

Keywords: Alzheimer's disease, Touchscreen, Amyloid beta, Early stage, App-KI

\section{Introduction}

The advancement of modern medical science has increased life expectancy and led to an increase in the aging population. As a result, age-related diseases are

\footnotetext{
*Correspondence: hmizoguchi@med.nagoya-u.ac.jp

${ }^{3}$ Department of Neuropsychopharmacology and Hospital Pharmacy,

Nagoya University Graduate School of Medicine, Nagoya, Aichi 466-8560, Japan

Full list of author information is available at the end of the article
}

becoming much more common. One of the most common age-related neurodegenerative disorders is Alzheimer's disease $(\mathrm{AD})$. This debilitating disease is mainly caused by accumulation of extracellular $A \beta$ and intracellular neurofibrillary tau tangles. Emerging data suggest that the disease process begins years before clinical diagnosis. The disease has a long preclinical phase with no clinical symptoms, followed by an early phase, also known as mild cognitive impairment phase, associated with mild symptoms, and a disease phase, when cognitive original author(s) and the source, provide a link to the Creative Commons licence, and indicate if changes were made. The images or other third party material in this article are included in the article's Creative Commons licence, unless indicated otherwise in a credit line to the material. If material is not included in the article's Creative Commons licence and your intended use is not permitted by statutory regulation or exceeds the permitted use, you will need to obtain permission directly from the copyright holder. To view a copy of this licence, visit http://creativecommons.org/licenses/by/4.0/. The Creative Commons Public Domain Dedication waiver (http://creativeco mmons.org/publicdomain/zero/1.0/) applies to the data made available in this article, unless otherwise stated in a credit line to the data. 
impairment becomes evident [1-3]. Early detection of the disease is important for effective intervention, including counseling, cognitive training, and medication [4]. Clinical studies have shown that the benefit of currently available medications is higher when initiated in the early phase of the disease $[5,6]$.

Basic AD research relies largely on various transgenic mouse models that experience accelerated accumulation of $A \beta$ and tau tangles. We have also demonstrated the mechanism of cognitive impairment in an animal model of $\mathrm{AD}$ and provided an effective approach for treatment of $\mathrm{AD}$ [7-10]; however, these mice exhibit artificial phenotypes and pathologies that are not present in human $\mathrm{AD}[11,12]$. To overcome these undesired phenotypes, we decided to utilize $A p p^{N L-G-F / N L-G-F}$ (App-KI) mice, a new $A D$ mouse model that overproduces $A \beta_{42}$ without overexpressing amyloid precursor protein (APP) [13]. $A p p^{N L-G-F}$ mice were generated by introducing three familial $\mathrm{AD}$-associated mutations at the endogenous mouse App locus by the knock-in approach [13]. App-KI mice exhibit aggressive $A \beta$ plaque deposition in the cortical and hippocampal regions at the age of 4 months.

Non-invasive cognitive tests are useful tools for identifying subjects with a high risk of developing symptomatic AD. Although App-KI mice have been subjected to various classical behavioral tasks to evaluate various cognitive parameters, detection of cognitive impairment at the earliest stage has been a challenge [14-17]. Moreover, the majority of these tests have low translational value because the testing conditions differ from those used for patients with AD. Recently, touchscreen-based behavioral testing systems have been developed that employ similar stimuli (images displayed on the screen) and reactions (touch) to assess performance, thereby ensuring an analogous testing system for humans and other disease model [18]. Hence, in this study we sought to detect cognitive impairments of $A p p$-KI mice associated with the early stage of $\mathrm{AD}$ in touchscreen-based behavioral tasks. We selected behavioral tests that require cortical and/or hippocampal function, as these regions are affected at the earliest stage in $A p p$-KI mice. Our results revealed that hippocampus-dependent touchscreen-based behavioral tasks could detect $\mathrm{AD}$ at an early stage for the first time, and also confirm the potential use of these cognitive tests in developing therapeutic approaches for preventing $\mathrm{AD}$ progression.

\section{Materials and methods}

\section{Animals}

Generation of $A p p$-KI $\left(A p p^{N L-G-F / N L-G-F}\right)$ mice was described previously [13]. Mice were obtained from the RIKEN Center for Brain Science (Wako, Japan). Male $A p p^{N L-G-F / N L-G-F}$, littermate, and wild-type (C57BL/6 J,
CLEA Japan, Inc., Japan, RRID:IMSR_JAX:000,664) mice were 4 months old at the start of touchscreen experiments and 6 months old for the Morris water maze test. All mice were housed in plastic cages and kept in a regulated environment $\left(23 \pm 1{ }^{\circ} \mathrm{C} ; 50 \pm 5 \%\right.$ humidity) with a 12-h light/dark cycle (lights on at 8:00 AM). Food (CE-2; CLEA Japan, Inc.) and tap water were available ad libitum. All experiments were performed in accordance with the Guidelines for Animal Experiments of Nagoya University, the Guiding Principles for the Care and Use of Laboratory Animals approved by the Japanese Pharmacological Society and the United States National Institutes of Health Guide for the Care and Use of Laboratory Animals. All experimental procedures were approved by the Institutional Animal Care and Use Committee of the Research Institute of Environmental Medicine, Nagoya University (Permit Number: RIEM19273). One week before starting behavioral experiments, mice were foodrestricted to achieve approximately $85 \%$ of their ad libitum bodyweight. Mice that exhibited severe bodily injury due to fighting were excluded from behavioral analysis.

\section{Touchscreen apparatus}

Touchscreen tests were performed as previously described with slight modifications [19-21]. Testing was conducted with a touchscreen-based automated operant system for mice housed within a sound- and light-attenuating box $(87 \times 50 \times 79 \mathrm{~cm}$, TOP-M1, O'hara \& Co., Ltd., Tokyo, Japan). The sound-attenuating box contained a house light, a ventilation fan that also provided white noise, and a pair of tone generators. The operant system contained a 15-inch touch panel unit and a 10-mg food pellet dispenser on the opposite side, fitted with a photocell head entry detector and a camera directly on top of the chamber. To decrease the frequency of unintended responses to the touchscreen due to contact with the tail or other body parts, a black plastic "mask" with task-specific response windows was placed in front of the screen: six windows for LD, two windows for visual discrimination and reversal learning, and three windows for different object-location paired-associate learning (dPAL) touchscreen-based behavioral tasks.

\section{Pretraining}

Before performing any of the touchscreen tests described in this study, mice had to go through pre-training steps, which consisted of the following: (1) Magazine training ( 1 day, $30 \mathrm{~min}$ ), in which mice received food (10-mg pellet, AIN-76A Rodent Tablet $10 \mathrm{mg}$, \#1811213 (5TUL), TestDiet, USA) for head entry into the food receptacle. (2) Autoshaping (1 day, $30 \mathrm{~min}$ ), in which food was delivered after contingent disappearance of presentation of a white stimulus in all windows. (3) Must touch (2 days, 
$60 \mathrm{~min} / 100$ trials), in which mice had to touch the screen to receive a food reward; all windows presented white stimuli; (4) Correct touch (2 days, $60 \mathrm{~min} / 100$ trials), in which mice had to touch the stimulus presented randomly in only one window to receive a food pellet; incorrect response had no effect. (5) Correct touch error ( 2 days, $60 \mathrm{~min} / 100$ trials, at least $80 \%$ correct response), in which mice had to correctly touch the randomly presented white stimulus to receive a food reward. Pretraining required 10 sessions and data from pretraining sessions are not shown.

In all pretraining steps and touchscreen tests, the trial was automatically started followed by a 3-s inter-trial interval (ITI), after which the mouse was required to enter its head into the food receptacle to start the trial, as described previously [21]. Head entry into the magazine during a session resulted in stimuli being displayed. A stimulus remained on the screen until the mouse responded to it, after which (if the choice was correct) the mouse was rewarded with a pellet accompanied by a tone, the magazine light was illuminated, and the trial was ended. This was followed by a 3-s ITI before starting the next trial. The house light was on during the trial. After a choice was made, the first head entry into the magazine after the ITI resulted in the stimuli being displayed for the next trial. This meant that on every trial, the mouse was situated at the back of the testing chamber when the stimuli were displayed.

\section{Location discrimination (LD) task}

The LD task was performed as described previously, with slight modification [22]. We used mask and stimulus dimensions as follows: number of windows, 6; window size, $25 \times 25 \mathrm{~mm}^{2}$, window gaps, $10 \mathrm{~mm}$; floor gap, $25 \mathrm{~mm}$; stimulus size, $25 \times 25 \mathrm{~mm}^{2}$. Following pretraining sessions, the LD task included an additional training step in which the mice were presented with two square white stimuli separated by an intermediate degree of separation (LDmedium: LDm). One square was designated as correct, and the other as incorrect (Phase 1). Responses at the correct location resulted in a reward delivery followed by 3-s ITI as described above. Responses at the incorrect location resulted in a 5-s timeout period with the house light off. Seven correct responses out of eight consecutive trials resulted in reversal of reward contingences, in which the previous incorrect location now became correct (Phase 2) (Fig. 1a). The initial correct location was counter balanced between animals in each genotype. Mice were given a maximum of 62 trials/session/d for 10 days. Following LDm training, pattern separation was assessed by presenting stimuli with either an LDlarge (LDl) task with a high degree of separation (four empty windows between two stimuli) or an
LDsmall (LDs) task with a low degree of separation (no empty windows between the two stimuli). Mice received a 30-min once daily session. Mice were subjected to each task for 4 days. The same degree of separation was presented for two consecutive days. The order of separation was counterbalanced between animals in each group across days. The average number of changes between phases during the LDl and LDs tasks were calculated. The LD task required 20 training sessions: 10 sessions for the LDm task and 10 sessions for the LDs and LDl tasks.

\section{Different object-location paired-associate learning (dPAL) task}

We used mask and stimulus dimensions as follows: number of windows, 3 ; window size, $57 \times 57 \mathrm{~mm}^{2}$; window gaps, $10 \mathrm{~mm}$; floor gap, $25 \mathrm{~mm}$; stimulus size, $53 \times 53 \mathrm{~mm}^{2}$. Following pretraining sessions, dPAL tasks also had one additional training step in which place-associated stimuli, were presented but the incorrect response had no effect. The dPAL experiment was performed as previously described [23]. Briefly, six different combinations were designed using three lined stimuli. Each stimulus was considered $\mathrm{S}^{+}$in a specific location. For each trial type, one visual stimulus was presented in its correct location and a second visual stimulus was presented in an incorrect location, leaving one window blank (Fig. 2a). Each combination of stimuli was presented an equal number of times. A correct choice was followed by reward delivery with tone, illumination of the magazine light, a 3-s ITI, and the next trial. An incorrect choice was followed by a 5-s timeout and a 3-s ITI, after which correction trials started in which the same set of stimuli are presented until the correct choice was made. Correction trials were not included in the calculation of percent correct response. The session finished either when the 120 trials were completed or when $60 \mathrm{~min}$ had passed. The dPAL task required 40 training sessions.

\section{Visual discrimination, and reversal learning task}

Acquisition Visual discrimination and reversal learning tests were performed as described previously [24] with slight modifications. We used mask and stimulus dimensions as follows: number of windows, 2; window size, $60 \times 60 \mathrm{~mm}^{2}$; window gap, $30 \mathrm{~mm}$; floor gap, $25 \mathrm{~mm}$; stimulus size, $60 \times 60 \mathrm{~mm}^{2}$. Mice were presented with a pair of black-and-white, brightness-matched stimuli on the touchscreen, one of which was correct $\left(\mathrm{S}^{+}\right)$and the other incorrect $\left(\mathrm{S}^{-}\right.$) (Fig. 3a). Response to the $\mathrm{S}^{+}$resulted in a tone, magazine illumination, and delivery of a single reward pellet. After incorrect responses, the house light was extinguished. Both correct and incorrect responses were followed by a 3-s ITI. Each daily session consisted of 100 trials in $60 \mathrm{~min}$. Percent correct response per 


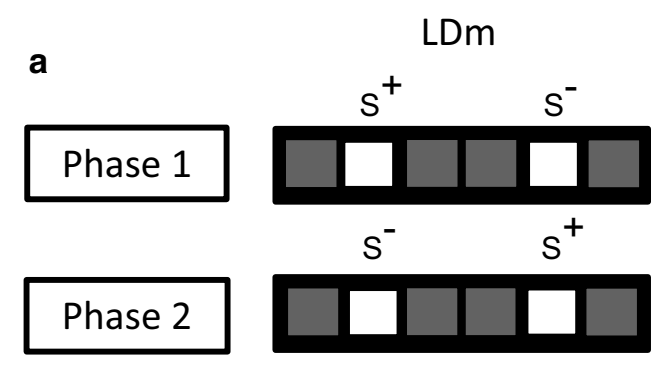

b

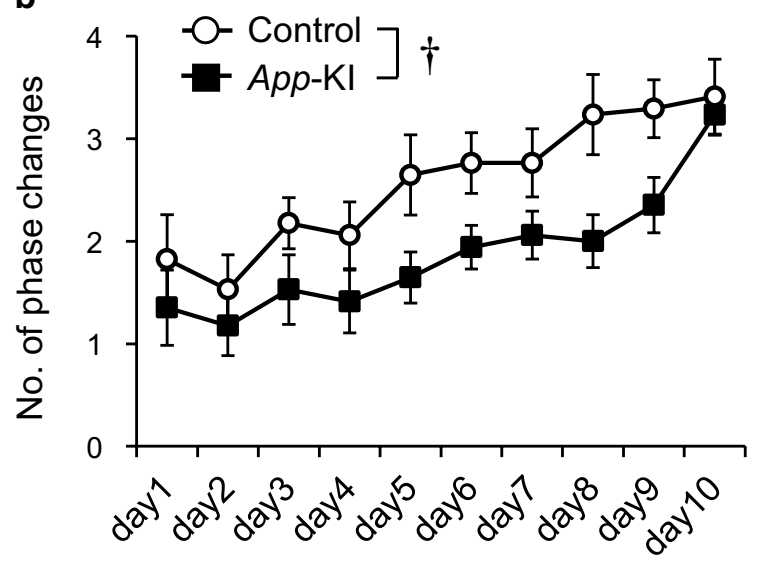

\section{Phase 1}

7 correct responses out of 8 trials

Phase 2

C
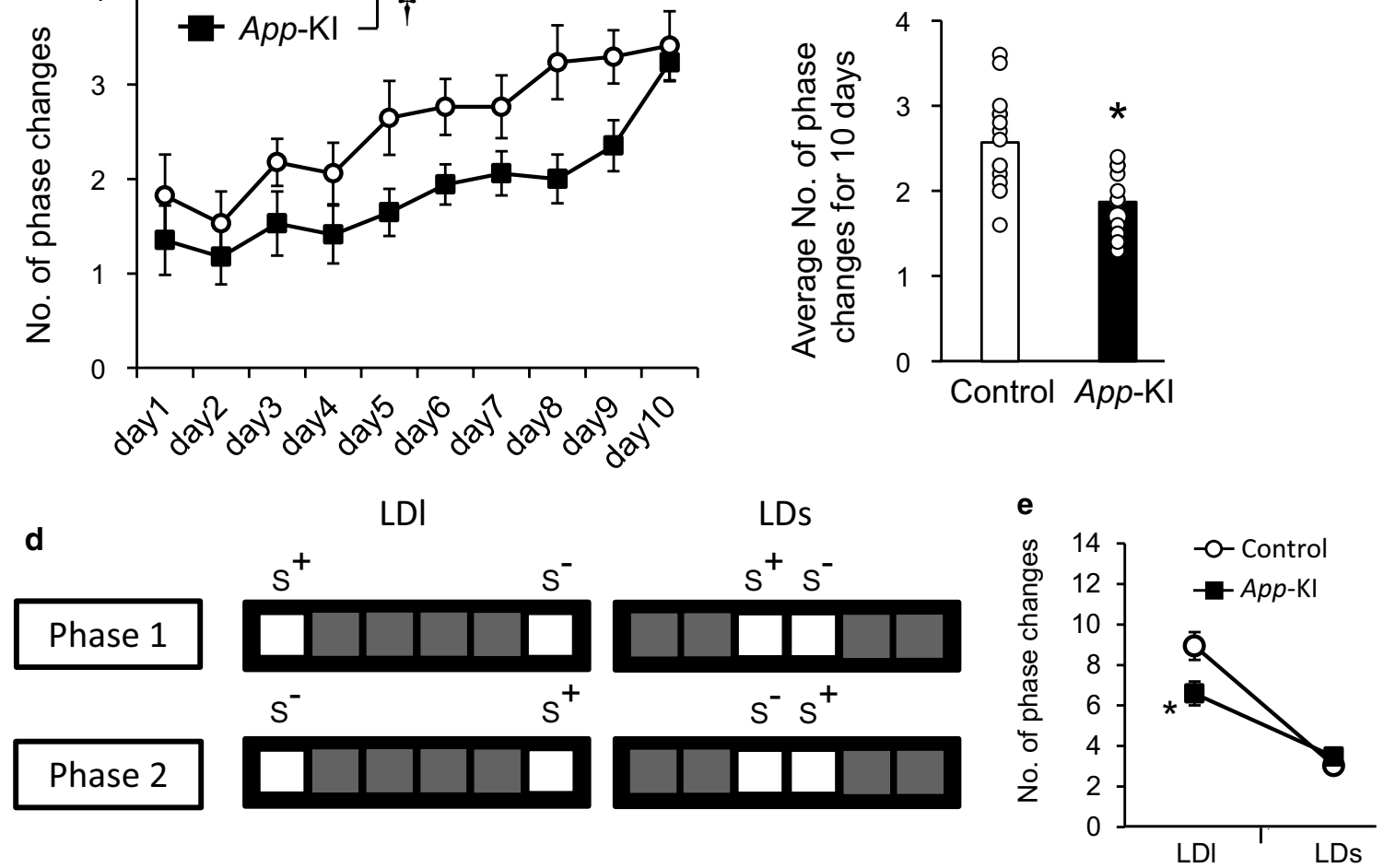

Fig. 1 Impaired pattern separation of App-KI mice in the LD task. a Diagram represents location discrimination tasks with a medium degree of separation between stimuli, as well as criteria for phase change. $\mathbf{b}, \mathbf{c}$, Number of phase changes in LDm training sessions in App-KI mice ( $\mathrm{n}=17)$. Mice were given a maximum of 62 trials/session/d for 10 days. Data are presented as means \pm SEM. ${ }^{*},+p<0.05$ vs control. $\mathbf{d}$ Diagram represents location discrimination tasks with high and low degree of separation between stimuli, as well as criteria for phase change. e Number of phase changes during 30-min training sessions in App-KI mice $(n=17)$. Data are presented as means \pm SEM. ${ }^{*} p<0.05$ vs LDI in control

100-trial session was calculated to evaluate performance. In this stage, although the animal reached an $80 \%$ correct response rate by the $5^{\text {th }}$ training session, we continued training to ensure that both groups of animals reached the highest level of learning.

Reversal After reaching the acquisition criteria, in following sessions, reward contingencies of $\mathrm{S}^{+}$and $\mathrm{S}^{-}$were reversed (Fig. 3a). In this phase, the previously unrewarded stimulus provided reward. Each training session had parameters similar to those in acquisition phase. The reversal phase continued until one or both groups reached $\sim 80 \%$ correct responses for 2 consecutive days, which required 8 sessions.

\section{Morris water maze test}

The Morris water maze test was performed as previously described [8] with minor modifications. Briefly, a circular pool $1.2 \mathrm{~m}$ in diameter was filled with water at a temperature of $22 \pm 1{ }^{\circ} \mathrm{C}$. A transparent platform $(7 \mathrm{~cm}$ in diameter) was submerged inside the pool. Objects of different shapes were placed on the surrounding walls. The mice were trained in three $60 \mathrm{~s}$ sessions for 9 days, 

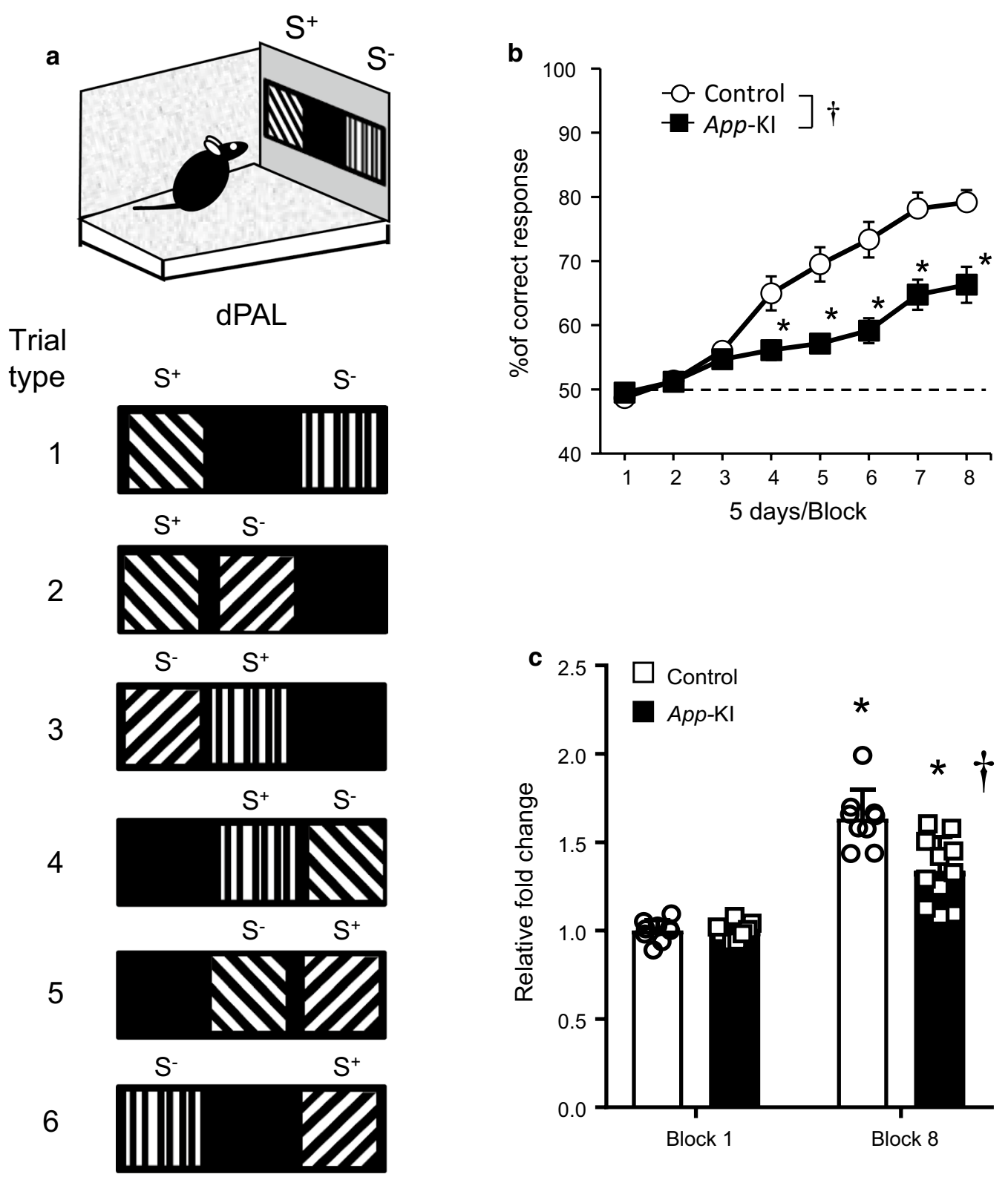

Fig. 2 Impaired associative memory of App-KI mice in the dPAL task. a Diagram represents all possible combinations of stimuli presentation. $\mathbf{b}$ Five-day blocks of performance during acquisition of the dPAL task. App-KI mice exhibited significantly worse performance than wild-type mice. $t p<0.05$ vs control. ${ }^{*} p<0.05$ vs each block in control. c Fold change relative to the first block. A significant difference was observed in block 8 , although both groups of mice exhibited significant improvement in the last data point relative to the first data point. $+p<0.05 \mathrm{vs}$ control in block 8 . ${ }^{*} p<0.05$ vs block 1 in each group. Data are presented as means \pm SEM $(n=9-11)$. Chance performance $(50 \%)$ is indicated by dashed lines

during which the platform and the objects on the walls were fixed in the same position. Twenty-four hours after the last training trial, the mice were given a probe test without the platform and were allowed to search the platform for $60 \mathrm{~s}$. Mice that did not swim were excluded from all behavioral experiments. The time taken to locate the escape platform (escape latency) and the distance moved was determined in each trial using the SMART system
(SMARTBASIC / SMART 3.0 BASIC PACK, Panlab, Barcelona, Spain). Nine training sessions were required to reach stable performance. Mice that exhibited odd behaviors such as spinning, lack of swimming, staying close to the periphery, or being unable to find the platform before time ran out after 6 days of training in the Morris water maze test were excluded from all behavioral experiments. 
a

Visual discrimination

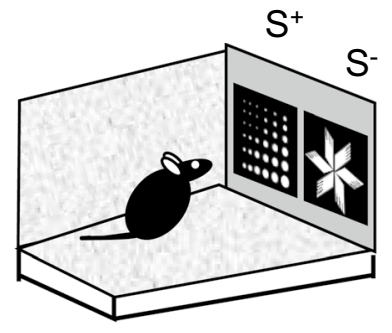

Reversal learning

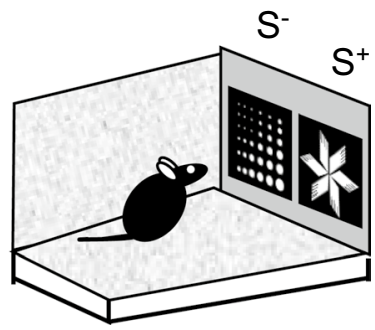

\section{b}
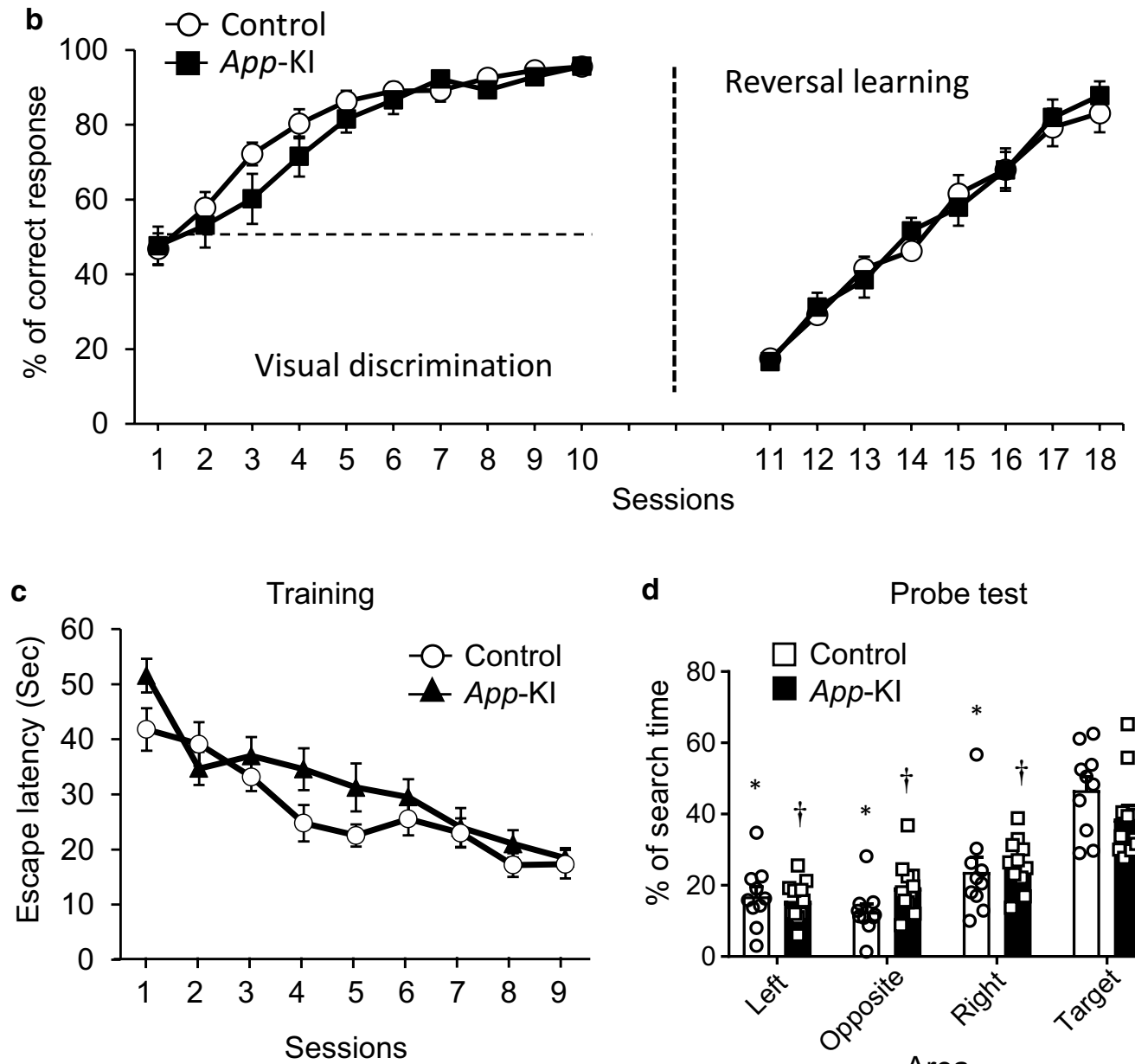

d

Probe test

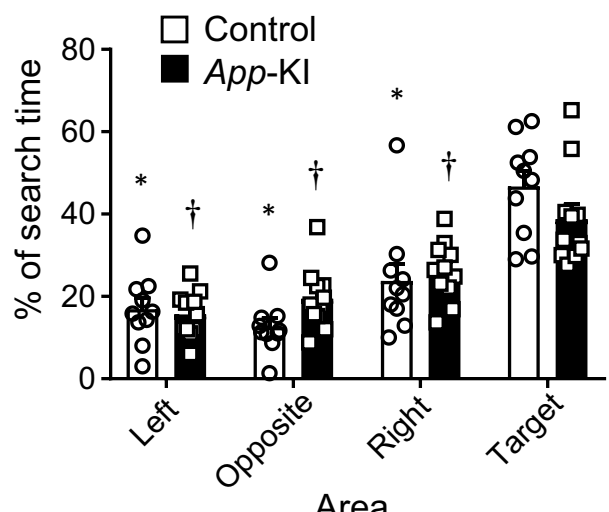

Fig. 3 App-KI mice exhibit no changes in cognitive flexibility or reference memory. $\mathbf{a}$, $\mathbf{b}$ Visual discrimination, reversal learning in the touchscreen-based operant system. a Diagram representing visual discrimination and reversal learning. $\mathbf{b}$ Behavioral performance of 4-5-month-old mice in both tasks. Data are presented as means \pm SEM ( $n=10-11)$. Chance performance (50\%) is indicated by dashed lines. $\mathbf{c}$, $\mathbf{d}$ Reference memory in Morris water maze test. c In 6-month-old mice, escape latency was measured during a 60-s session in the water maze test. d Spatial memory of a platform's location during the training phase was tested in the probe test. Percentage of time spent in each quadrant was measured. Data are presented as means \pm SEM $(n=10-11)$. $p<0.05$ vs target in WT mice (Tukey test for multigroup comparisons). $+p<0.05$ v target in App-KI mice (Tukey test for multigroup comparisons)

\section{Immunohistochemistry}

Mice were deeply anesthetized by high-concentration isoflurane for animal (MSD Animal Health K.K., Tokyo,
Japan) and perfused with $4 \%$ paraformaldehyde in phosphate buffer (4\% PFA). Brains were dissected and postfixed with $4 \%$ PFA for $24 \mathrm{~h}$, and then cryoprotected in 
$30 \%$ sucrose in PBS for $24 \mathrm{~h}$. Twelve-micron sagittal cryosections were prepared and treated with HistoVT One (Nacalai Tesque, Japan) at $70{ }^{\circ} \mathrm{C}$ for $20 \mathrm{~min}$. The sections were then pre-incubated with $5 \%$ normal donkey serum/0.3\% Triton-X-100 in PBS for $1 \mathrm{~h}$ and immunostained with primary antibodies against Iba-1 (1:250, Novus Biologicals, USA, RRID:AB_521594), GFAP (1:500, DAKO, Denmark, RRID:AB_10013382), and the $\mathrm{N}$-terminal region of human $\mathrm{A} \beta$ conjugated with biotin (1:200, IBL, Japan, RRID:AB_10705565) followed by Alexa Fluor 488-conjugated anti-goat IgGs (1:1000, Invitrogen, USA, RRID:AB_2534102), Alexa Fluor 647conjugated anti-rabbit IgGs (1:500, Jackson ImmunoResearch Laboratories, USA, RRID:AB_2492288), and Alexa Fluor 546-conjugated streptavidin (1:500, Invitrogen, USA, RRID:AB_2532130). Images were obtained on a confocal laser microscope (LSM700, Carl-Zeiss, Germany).

Immunostaining for analysis of hippocampal neurogenesis was performed as described previously with slight modifications [25]. Twenty-five-micron coronal frozen sections were post fixed with 4\% PFA for $20 \mathrm{~min}$ and washed three times with PBS. They were incubated with methanol for $30 \mathrm{~min}$, and then with $0.3 \%$ Triton$\mathrm{X} / \mathrm{PBS}$ buffer for $30 \mathrm{~min}$ at $37{ }^{\circ} \mathrm{C}$. They were then autoclaved with Antigen Unmasking Solution (H-3300, Sigma-Aldrich, RRID:AB_2336226) at $105^{\circ} \mathrm{C}$ for $2 \mathrm{~min}$, followed by three washes with PBS. They were incubated for $30 \mathrm{~min}$ in blocking serum (10\% normal goat serum in $0.3 \%$ Triton-X $100 / \mathrm{PBS}$ ) and then for $24 \mathrm{~h}$ at $4{ }^{\circ} \mathrm{C}$ in the presence of a primary antibody against doublecortin (DCX) (E-6) (a neuronal lineage marker) (1:100, sc-271390, Santa Cruz Biotechnology, Dallas, TX, USA, RRID:AB_10610966) and Ki-67 (SP6) (a proliferating cell marker) (1:100, ab16667, Abcam, Cambridge, MA, USA, RRID:AB_302459). Sections were then washed three times with $0.05 \%$ tween in PBS, incubated in a secondary antibody (Alexa 488, RRID:AB_143165 and Alexa 546, RRID:AB_144695) (1:1000, Invitrogen, USA) for $2 \mathrm{~h}$, and washed three times with $0.05 \%$ Tween in PBS. Antibodies were diluted in the Can Get Signal ${ }^{\circledR}$ immunostain Solution A (NKB-501, TOYOBO, Japan).

The dentate gyrus (DG) was segregated into dorsal regions (approximately -1.8 to $-2.3 \mathrm{~mm}$ from bregma) [26], and cells in each segregation were quantified to determine any difference in neurogenesis between groups. Samples were observed with a microscope (BZ9000, KEYENCE Corp., Osaka, Japan) and analyzed at $40 \times$ magnification. The number and density of cells positive for immunoreactivities were analyzed using ImageJ. The values were summed and divided by the number of slices analyzed for each animal. Four areas of interest $(362.99 \mu \mathrm{m} \times 273.31 \mu \mathrm{m})$, two each in the right and left
DG, were imaged on one slice, and averages of at least five slices (20 areas) in each mouse were counted within areas of interest and used for statistical analysis.

\section{Statistical analysis}

All data are expressed as means \pm SEM. Statistical analyses were performed with GraphPad Prism 7 (GraphPad Software, San Diego, CA, USA, RRID:SCR_002798). Statistical significance $(\mathrm{p}<0.05)$ was determined using Student's $t$-test for comparisons between two groups; two-way analysis of variance (ANOVA) for multigroup comparisons; or repeated-measures ANOVA. Bonferroni test and Tukey test were used for post hoc comparison when the F value was significant. The sample size for each experiment was determined based on our previous studies using the relevant type of experiment $[7,8,27]$.

\section{Results}

The LD task is a useful method for detecting impairment in pattern separation in App-KI mice at the early stage We subjected 4-5-month-old App-KI mice to the LD task. In the LDm training sessions, mice were given a maximum of 62 trials/session/d for 10 days. Statistical analysis revealed a significant difference between the two groups [Fig. 1b, two-way ANOVA with repeated measures, group, $\mathrm{F}(1,32)=20.22$, $\mathrm{p}<0.0001$, day, $\mathrm{F}(9$, $288)=15.16, \mathrm{p}<0.0001$, interaction, $\mathrm{F}(9,288)=1.03$, $\mathrm{p}=0.4157$; Fig. $1 \mathrm{c}, \mathrm{t}(32)=4.497, \mathrm{p}<0.0001$ by $t$-test], indicating that $A p p$-KI mice exhibited poorer performance than the control group. On day 10, we observed the same level of performance between the two groups in $\mathrm{LDm}$ training. Following $\mathrm{LDm}$ training, mice performed both LDl and LDs tasks. We observed a significant difference in behavioral performance only in the LDl task, whereas both groups of mice performed similarly in the LDs task [Fig. 1e, two-way ANOVA followed by Tukey test, group, $\mathrm{F}(1,64)=3.305, \mathrm{p}=0.0738$, task, $\mathrm{F}(1,64)=75.99, \mathrm{p}<0.0001$, interaction, $\mathrm{F}(1,64)=7.791$, $\mathrm{p}=0.0069]$.

\section{The dPAL task is a useful method for detecting impairment} in associative memory in App-KI mice at the early stage

Because $A p p$-KI mice exhibited behavioral impairment in the hippocampus-dependent pattern separation in the LD task, we sought to assess their performance in other hippocampus-dependent tasks. Therefore, we next subjected $A p p$-KI mice to the dPAL task. In this task, animals learn to associate three different stimuli with their correct spatial locations (Fig. 2a). Learning performances from five sessions were combined to yield a single data point. Relative to the control group, $A p p$-KI mice exhibited significantly impaired learning [Fig. 2b, two-way ANOVA with repeated measure followed by Bonferroni 
test, group, $F(1,18)=16.67, \mathrm{p}=0.0007$, block, $\mathrm{F}(7$, $126)=78.00, \mathrm{p}<0.0001$, interaction, $\mathrm{F}(7,126)=10.46$, $\mathrm{p}<0.0001)$. Especially on blocks $4-8$, there were significant differences between two groups. Moreover, when fold change relative to the first block was calculated, both groups exhibited significant improvement in block 8 [Fig. 2c, two-way ANOVA with repeated measure followed by Bonferroni test, group, $F(1,18)=16.37$, $\mathrm{p}=0.0008$, block, $\mathrm{F}(1,18)=109.3, \mathrm{p}<0.0001$, interaction, $\mathrm{F}(1,18)=9.981, \mathrm{p}=0.0054]$.

\section{Visual discrimination and reversal learning tasks cannot detect cognitive impairment in App-KI mice at the early stage}

We also subjected 4-5-month-old App-KI mice to a visual discrimination and reversal task. During the acquisition phase, animals learned to discriminate between the reward contingent and non-contingent stimuli. In the acquisition phase, general ability to distinguish visual stimuli was assessed. In the reversal phase, cognitive flexibility was required to inhibit the previously learned stimulus-reward association and relearn a new contingency for a familiar stimulus. In both the acquisition and reversal phase, App-KI mice performed similarly to the control group [Fig. 3b, visual discrimination: two-way ANOVA with repeated measure, group, $\mathrm{F}(1$, $19)=0.9875, \mathrm{p}=0.3328$, day, $\mathrm{F}(9,171)=76.41, \mathrm{p}<0.0001$, interaction, $\mathrm{F}(9,171)=1.322, \mathrm{p}=0.2286$; reversal learning: two-way ANOVA with repeated measure, group, $\mathrm{F}(1,19)=0.06718, \mathrm{p}=0.7983$, day, $\mathrm{F}(7,133)=93.47$, $\mathrm{p}<0.0001$, interaction, $\mathrm{F}(7,133)=0.4563, \mathrm{p}=0.8644]$.

\section{The Morris water maze test cannot detect impairment in spatial reference memory in App-KI mice at the early stage}

We also evaluated the reference memory of App-KI mice at the age of 6 months using the Morris water maze test. At this age, both groups of mice exhibited similar performance in learning the position of the hidden platform [Fig. 3c, two-way ANOVA with repeated measure, group, $\mathrm{F}(1,19)=3.924, \mathrm{p}=0.0623$, day, $\mathrm{F}(8,152)=19.53$, $\mathrm{p}<0.0001$, interaction, $\mathrm{F}(8,152)=1.295, \mathrm{p}=0.2501]$. Following the training phase, mice were subjected to the probe test, in which the hidden platform was removed. Both groups of mice spent the largest amount of time in the target quadrant where the platform was previously positioned. Although App-KI mice spent less time than control mice in the target quadrant, the difference was not significant [Fig. 3d, two-way ANOVA, group, $\mathrm{F}(1,76)=1.233 \times 10^{-8}, \mathrm{p}>0.9999$, area, $\mathrm{F}(3,76)=37.47$, $\mathrm{p}<0.0001$, interaction, $\mathrm{F}(3,76)=2.312, \mathrm{p}=0.0828]$.
$A \beta$ plaque deposition, glia accumulation, and impaired adult neurogenesis are much more advanced in App-KI mice at 6 months

Finally, we examined $\mathrm{A} \beta$ plaque deposition in AppKI mice at the age of 2, 4, and 6 months. $A \beta$ plaques increased with age in the cortices and hippocampi of $A p p$-KI mice, and were first observed around 4 months of age. Iba-1- and GFAP-positive cells also accumulated along with $A \beta$ plaques (Fig. 4a). Moreover, we observed the age-dependent presence of activated microglia and GFAP-reactive astrocytes around $A \beta$ plaques (Fig. 4a).

In addition, we examined adult neurogenesis in hippocampi of App-KI mice. Cells in the DG positive for DCX, a marker of newborn neurons, were less abundant in App-KI mice than in wild-type mice [Fig. 4b, c, $\mathrm{t}(6)=3.126, \mathrm{p}=0.0204$ by $t$-test]. Cells positive for Ki-67, a proliferation marker, were also less abundant in App-KI mice at the same age [Fig. $4 \mathrm{~b}, \mathrm{~d}, \mathrm{t}(6)=3.07, \mathrm{p}=0.0219$ by $t$-test]. Taken together, these data suggest that accumulation of glia and impaired adult neurogenesis were accompanied by A $\beta$ plaque expression in $A p p-\mathrm{KI}$ mice at 6 months.

\section{Discussion}

In this study, we used behavioral tests that required proper function of hippocampal and cortical regions [24, $28,29]$. These tasks assess different parameters of cognitive function. The LD task measures pattern separation, the dPAL task assesses object-in-place associative memory, the Morris water maze test assesses spatial reference memory, and the visual discrimination and reversal learning tasks measure cognitive flexibility [8, 23-25]. This is the first study to evaluate cognitive functions in AD model mice using a systematic touchscreen-based behavioral test battery.

We used a touchscreen-based LD task to analyze the pattern separation ability of AD model mice. We utilized an App-KI mouse model that exhibits significant $\mathrm{A} \beta$ accumulation, increased neuroinflammation, and synaptic alteration in the hippocampus starting at the age of 4 months without overproducing other APP fragments such as the soluble fragment of APP, C-terminal fragment- $\beta$, C-terminal fragment- $\alpha$, or APP intracellular domain [11-13]. App-KI mice do not exhibit tau pathology, neurodegeneration, or severe neuron loss, suggesting that they are models of preclinical AD [12]. In these mice, $A \beta$ accumulation in the cortical region starts even earlier (at 2 months) [13]. We also observed a similar pattern of $A \beta$ accumulation (Fig. 4a). In addition, we detected the age-dependent presence of activated microglia and GFAP-reactive astrocytes around $A \beta$ plaques, starting at 4 months, indicating that 
a
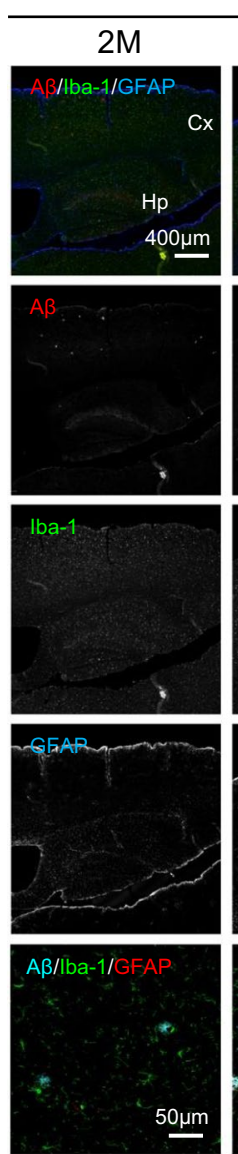

App-KI mouse

$4 \mathrm{M}$
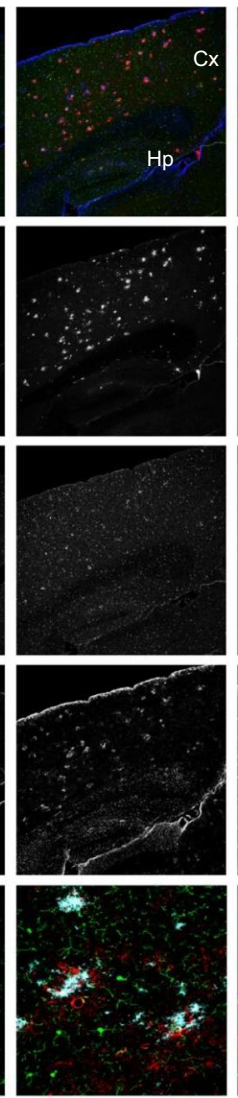

b
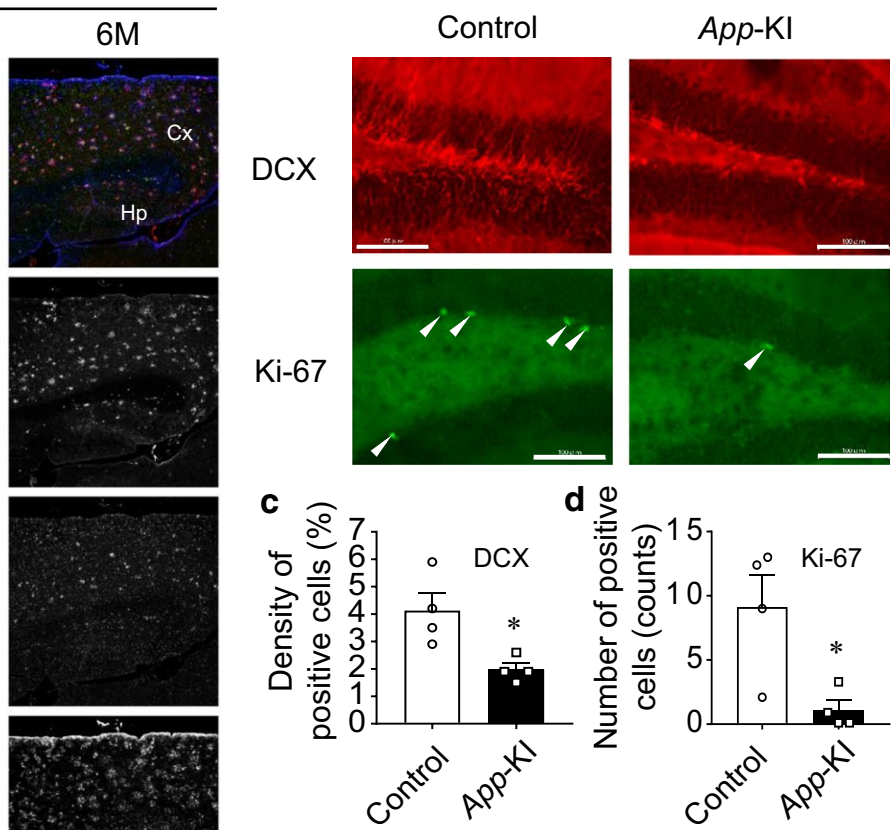

Fig. 4 Representative images of amyloid plaque, microglia, and astrocyte expression in App-KI mice. a Upper panel shows localization of $A \beta$ (red), Iba-1 (green), and GFAP (blue) in the cortico-hippocampus of App-KI at 2-6 months age. These are followed by individual images of A GFAP (from top to bottom). Bottom panels are representative higher-magnification images of activated microglia and GFAP-reactive astrocytes around A $\beta$ plaques. These panels (Merge) show localization of A $\beta$ (light blue), Iba-1 (green), and GFAP (red) in the cortico-hippocampus of App-KI at 2-6 months of age. $\mathbf{b}-\mathbf{d}$ Adult neurogenesis in hippocampus of App-KI mice. $\mathbf{b}$ Representative images of DCX and Ki-67 expression. (Scale bar: $100 \mu \mathrm{m})$ c Quantitative data of DCX-positive cells. Expression levels were reduced in the hippocampus of App-KI mice. d Quantitation of Ki-67positive cells. Ki-67-positive cells (arrowheads) were less abundant in hippocampus of App-KI mice. Data are presented as means $\pm S E M(n=4)$. ${ }^{*} \mathrm{p}<0.05$ (t-test)

amyloidosis-associated neuroinflammation occurred at an early stage in App-KI mice (Fig. 4a) [30]. Although many researchers have subjected these mice to different classical behavioral tests, behavioral impairments were not reported at the early stage (Table 1) [14-17]. However, using a touchscreen-based LD task in App-KI mice, we detected cognitive impairment around 4-5 months of age (Fig. 1). In particular, App-KI mice exhibited significant impairment when the stimuli were separated by a large gap (LDl task) (Fig. 1e). By contrast, in the LDs task, we observed no significant difference between the App$\mathrm{KI}$ and control groups. It is possible that the gap between stimuli in LDs is important, and it may have been too narrow for the mice to discriminate images. Indeed, in the LDm training, $A p p$-KI mice exhibited poorer performance than the control group (Fig. $1 \mathrm{~b}$ and c). At a minimum, these results imply that mild pathological changes had progressed at an early stage in App-KI mice. Further experiments are required to prove this hypothesis.

Pattern separation requires the function of complex neuronal networks in the hippocampal subregion (entorhinal cortex-DG-CA3-CA1 circuit) [31-33]. Previous studies showed that these subregions and networks are the primary targets of $A \beta$ pathology during the early stage of AD [34-37]. In App-KI mice, accumulation of $A \beta$ plaque is associated with loss of synaptic markers [13]. Moreover, cholinergic synapses are essential for survival, and glutamatergic receptor signaling is required for 
Table 1 Summary of significant differences in behavioral tests in App-KI mice in this study vs. previous studies

\begin{tabular}{|c|c|c|c|}
\hline Behavioral tests & Test significance & Significant difference & References \\
\hline Y-maze & Short-term memory & Yes (6 months) & Saito et al. [13] \\
\hline Place preference and reversal task & Spatial learning and reversal learning & Yes (13-14 months) & Masuda et al. [16] \\
\hline Serial reaction time task & Impulsivity and attention & Yes (13-14 months) & \\
\hline Place avoidance & Extinction learning & Yes (8-9 months) & \\
\hline Delay discounting task & Compulsive behavior & Yes (8-9 months) & \\
\hline Novel object recognition & Recognition memory & Not detected (6 months) & Whyte et al. [17] \\
\hline Y-maze & Working memory & Not detected (6 months) & \\
\hline Morris water maze & Spatial reference memory & Not detected (6 months) & \\
\hline Open field & Anxiety & Yes (6 months) & \\
\hline Fear conditioning & Fear learning & Not detected (15-18 months) & Sakakibara et al. [14] \\
\hline Elevated plus maze & Anxiety-related behavior & Yes (6-18 months) & \\
\hline Barnes maze & Spatial memory & Yes (8 months) & \\
\hline Spatial reversal learning & Flexibility and impulse control & Not detected (8 months) & \\
\hline Morris water maze & Spatial reference memory & Not detected (10-11 months) & Latif-Hernandez et al. [15] \\
\hline Cage activity and exploration & Spontaneous activity & Yes (3 and 10 months) & \\
\hline Social Preference Social Novelty (SPSN) test & Social memory & Not detected $(3,6$, and 10 months) & \\
\hline Location discrimination & Pattern separation & Yes (4-5 months) & Present study \\
\hline $\begin{array}{l}\text { Different object-location paired-associate } \\
\text { learning }\end{array}$ & Paired-associative memory & Yes (4-6 months) & \\
\hline Visual discrimination, Reversal learning & Cognitive flexibility & Not detected (4-5 months) & \\
\hline Morris water maze & Spatial reference memory & Not detected (6 months) & \\
\hline
\end{tabular}

proper migration and positioning of newborn neurons in DG of adult brain $[38,39]$, a physiological process that is essential for optimum pattern separation performance in rodents [40-42]. Pattern separation in human is also impaired at the early stage of $\mathrm{AD}[43,44]$ and has been linked to poor hippocampal neurogenesis [43, 45]. In this study, immunohistochemical analysis of both DCX and Ki-67 revealed altered adult hippocampal neurogenesis in 6-month-old App-KI mice (Fig. 4b). Notably, in this regard, adult hippocampal neurogenesis is impaired before the onset of classical AD pathology in AD mice [46]. Inflammation-mediated disruption of adult hippocampal neurogenesis impairs pattern separation [47]. Elevated levels of DCX-positive cells enhance performance in hippocampus-dependent tasks [48]. Based on these observations and together with our immunohistochemical data (Fig. 4), we speculate that the cognitive deficit observed in this study was due to $A \beta$-associated synaptic loss, which altered adult neurogenesis and proper incorporation of newborn cells into the hippocampal network. In fact, synaptic alterations have been reported in the same mouse model: specifically, presynaptic synaptophysin and postsynaptic PSD95 immunoreactivity near the $A \beta$ plaque is reduced [11]. Further research is needed to determine whether the cognitive impairments we observed in the tasks described above are the result of these synaptic alterations.
Next, we assessed the performance of $A p p$-KI mice in the dPAL task to assess paired-associate memory. Previous studies demonstrated that dPAL, as part of the Cambridge Neuropsychological Test Automated Battery (CANTAB), is very efficient at distinguishing early-stage patients with AD from those suffering cognitive impairment caused by other clinical conditions [49-51]. This task is also sensitive to dorsal hippocampal dysfunction and neurogenesis in DG [22, 29], and both of these factors are affected from the early stage of $\mathrm{AD}$ pathology $[36,37,52]$. In accordance with previous studies, our results revealed significant impairment in the App-KI group relative to control mice (Fig. 2b), although cognitive function related to object-in-place memory was maintained in the App-KI group (Fig. 2c). This result reinforces clinical data showing that the dPAL task provides a sensitive means for detecting $\mathrm{AD}$ at its early stage. Our findings are the first data to demonstrate the potential use of touchscreen-based LD and APAL tasks in detecting cognitive impairments associated with early AD. Moreover, it is possible that our test battery can detect impairment at an earlier age (2-4 months of age) than 4-6 months, because massive accumulation of amyloid $\beta$ and glial cells were observed in 4-month-old mice (Fig. 4a). We would like to continue the experiments for this point in future studies. 
We also assessed the behavioral performance of AppKI mice in touchscreen-based visual discrimination and reversal learning, as well as in the classical Morris water maze test (Fig. 3). Neither of these tests revealed any cognitive impairment at the age tested. However, a previous study reported significantly improved performance in TgCRND8 (4.5-months of age) mice during the reversal phase [24]. By contrast, APPSwDl/Nos2 $2^{-1-}$ (4-5 months of age) and APPS1-21 (6 months of age) mice exhibited impaired performance in the reversal learning task [53, 54]. Medial prefrontal cortex lesions facilitate reversal learning in mice [55], whereas lesions of the orbitofrontal cortex impair reversal learning $[56,57]$. It would be intriguing to find out whether different mutations deposit $\mathrm{A} \beta$ differentially in specific brain regions. Interestingly, in this study, App-KI mice performed similarly to the control group, even though significant $A \beta$ had accumulated in the cortical region at the age tested here. One possible reason for this discrepancy may be the number of trials per session. In this study, the number of trials in a single session was much higher than in the reports described above, which may have compensated for the mild impairment in behavioral flexibility at the early stage of AD. Another possible explanation for this result may be that at the early stage of $\mathrm{AD}$, the hippocampal function is more prone to $A \beta$-related dysfunction, whereas the cortical regions are more resilient and show impaired function at a much later stage. Therefore, hippocampus-dependent $\mathrm{LD}$ and dPAL tasks were impaired but not reversal learning, which depends on the cortical subregion. However, to rule out any involvement of cortical regions in pattern separation or associative learning, further experiments will be required.

In the Morris water maze test, App-KI mice exhibited slightly worse performance than the control group, but the difference was not statistically significant. Our result is in accordance with a recent study showing that App-KI mice performed similarly to the control group [17]. Executive function related to pattern separation and pairedassociate memory is impaired at the early stage of $\mathrm{AD}$, whereas reference memory is impaired at a later stage. Perhaps at the later stage of the disease (11-12 months), the subtle behavioral differences observed in our study may become significant. Moreover, mice usually avoid wet conditions and forced swimming stress may induce a stress tolerance-dependent bias in the outcome of the Morris water maze test. Rodent behavioral tests were historically developed using animals undaunted by wet conditions. In that sense, the touchscreen-based test battery would be superior.

In conclusion, using App-KI mice, which recapitulate $A \beta$ pathology without overexpression of APP fragments, we showed that hippocampus-dependent touchscreen-based tasks can detect AD-associated behavioral impairments with high sensitivity at the early stage of the disease when classical tests cannot efficiently assess cognitive impairment. The reason for the sensitive difference between behavioral tasks may be that the required cognitive function and the difficulty of the task differ between these tasks. These data suggest that touchscreen-based tasks could be useful for advancing the translational studies by evaluating the efficacy of candidate therapeutics in rodent models of AD from an early stage.

\section{Abbreviations \\ AD: Alzheimer's disease; A $\mathrm{B}$ : Amyloid beta; APP: Amyloid precursor protein; App-KI: $A p p^{N L-G-F / N L-G-F}$ Knock-in; CANTAB: Cambridge neuropsychological test automated battery; DCX: Doublecortin; DG: Dentate gyrus; PBS: Phos- phate buffer in saline; dPAL: Different paired-associate learning; ITI: Inter-trial interval; LD: Location discrimination.}

\section{Acknowledgements}

Not applicable.

\section{Authors' contributions}

Md.A.B.S. carried out and analyzed behavioral experiments. O.K., Y.D., and A.S carried out immunohistochemical experiments and analyzed the data. O.K., A.S., F.E., T.S., and T.C.S. bred and provided the mice. H.M. designed, carried out, and analyzed the experiments and collected and assembled the data with Md.A.B.S and K.F. H.M. and Md.A.B.S. drafted the manuscript with K.Y. All authors read and approved the final manuscript.

\section{Funding}

This work was partially supported by the following funding sources: a Grantin-Aid for Scientific Research (19H03532, 19H05017, 19K21811) from MEXT; a grant for biomedical research from the SRF, Japan; a grant from Takeda Science Foundation; a grant from the Mishima Kaiun Memorial Foundation; the Hori Sciences And Arts Foundation; and a grant from the Strategic Research Program for Brain Science from Japan Agency for Medical Research and Development (AMED) under Grant Number 20dm0107135h0005. All experiments were conducted in compliance with the ARRIVE guidelines.

\section{Availability of data and materials}

The data used in our study are available from the authors on reasonable request.

\section{Ethics approval and consent to participate}

All experimental procedures were approved by the Institutional Animal Care and Use Committee of the Research Institute of Environmental Medicine, Nagoya University (Permit Number: RIEM19273).

\section{Consent for publication}

Not applicable.

\section{Competing interests}

The authors declare that they have no competing interests.

\section{Author details}

${ }^{1}$ Research Center for Next-Generation Drug Development, Research Institute of Environmental Medicine, Nagoya University, Nagoya, Aichi 464-8601, Japan. ${ }^{2}$ Department of Neuroscience and Pathobiology, Research Institute of Environmental Medicine, Nagoya University, Nagoya, Aichi 464-8601, Japan. ${ }^{3}$ Department of Neuropsychopharmacology and Hospital Pharmacy, Nagoya University Graduate School of Medicine, Nagoya, Aichi 466-8560, Japan.

${ }^{4}$ Laboratory for Proteolytic Neuroscience, RIKEN Center for Brain Science, Wako, Saitama 351-0198, Japan. ${ }^{5}$ Department of Neurocognitive Science, Institute of Brain Science, Nagoya City University Graduate School of Medical Sciences, Nagoya, Aichi 467-8601, Japan. 
Received: 16 September 2020 Accepted: 28 October 2020

Published online: 13 November 2020

\section{References}

1. Albert MS, DeKosky ST, Dickson D, Dubois B, Feldman HH, Fox NC, et al. The diagnosis of mild cognitive impairment due to Alzheimer's disease: recommendations from the National Institute on Aging-Alzheimer's Association workgroups on diagnostic guidelines for Alzheimer's disease. Alzheimers Dement. 2011;7:270-9.

2. Jack CR Jr, Bennett DA, Blennow K, Carrillo MC, Dunn B, Haeberlein SB, et al. NIA-AA research framework: toward a biological definition of Alzheimer's disease. Alzheimers Dement. 2018;14:535-62.

3. Sperling RA, Aisen PS, Beckett LA, Bennett DA, Craft S, Fagan AM, et al. Toward defining the preclinical stages of Alzheimer's disease: recommendations from the National Institute on Aging-Alzheimer's Association workgroups on diagnostic guidelines for Alzheimer's disease. Alzheimers Dement. 2011;7:280-92.

4. De Roeck EE, Engelborghs S, Dierckx E. Next generation brain health depends on early Alzheimer disease diagnosis: from a timely diagnosis to future population screening. J Am Med Direct Assoc. 2016;17:452-3.

5. Doraiswamy PM, Krishnan KR, Anand R, Sohn H, Danyluk J, Hartman RD, et al. Long-term effects of rivastigmine in moderately severe Alzheimer's disease: does early initiation of therapy offer sustained benefits? Prog Neuropsychopharmacol Biol Psychiatry. 2002;26:705-12.

6. Farlow M, Anand R, Messina J Jr, Hartman R, Veach J. A 52-week study of the efficacy of rivastigmine in patients with mild to moderately severe Alzheimer's disease. Eur Neurol. 2000;44:236-41.

7. Fukumoto K, Mizoguchi H, Takeuchi H, Horiuchi H, Kawanokuchi J, Jin S, et al. Fingolimod increases brain-derived neurotrophic factor levels and ameliorates amyloid beta-induced memory impairment. Behav Brain Res. 2014;268:88-93.

8. Takeuchi H, Mizoguchi H, Doi Y, Jin S, Noda M, Liang J, et al. Blockade of gap junction hemichannel suppresses disease progression in mouse models of amyotrophic lateral sclerosis and Alzheimer's disease. PLoS ONE. 2011;6:e21108.

9. Mizuno T, Doi Y, Mizoguchi H, Jin S, Noda M, Sonobe Y, et al. Interleukin-34 selectively enhances the neuroprotective effects of microglia to attenuate oligomeric amyloid-beta neurotoxicity. Am J Pathol. 2011;179:2016-27.

10. Doi Y, Mizuno T, Maki Y, Jin S, Mizoguchi H, Ikeyama M, et al. Microglia activated with the toll-like receptor 9 ligand $\mathrm{CpG}$ attenuate oligomeric amyloid beta neurotoxicity in in vitro and in vivo models of Alzheimer's disease. Am J Pathol. 2009;175:2121-32.

11. Nilsson P, Saito T, Saido TC. New mouse model of Alzheimer's. ACS chemical neuroscience. 2014;5:499-502.

12. Sasaguri H, Nilsson P, Hashimoto S, Nagata K, Saito T, De Strooper B, et al. APP mouse models for Alzheimer's disease preclinical studies. EMBO J. 2017;36:2473-87.

13. Saito T, Matsuba Y, Mihira N, Takano J, Nilsson P, Itohara S, et al. Single App knock-in mouse models of Alzheimer's disease. Nat Neurosci. 2014;17:661-3.

14. Sakakibara Y, Sekiya M, Saito T, Saido TC, lijima KM. Cognitive and emotional alterations in App knock-in mouse models of Abeta amyloidosis. BMC Neurosci. 2018;19:46.

15. Latif-Hernandez A, Shah D, Craessaerts K, Saido T, Saito T, De Strooper B, et al. Subtle behavioral changes and increased prefrontal-hippocampal network synchronicity in APP(NL-G-F) mice before prominent plaque deposition. Behav Brain Res. 2019;364:431-41.

16. Masuda A, Kobayashi Y, Kogo N, Saito T, Saido TC, Itohara S. Cognitive deficits in single App knock-in mouse models. Neurobiol Learn Mem. 2016;135:73-82.

17. Whyte LS, Hemsley KM, Lau AA, Hassiotis S, Saito T, Saido TC, et al. Reduction in open field activity in the absence of memory deficits in the App(NL-G-F) knock-in mouse model of Alzheimer's disease. Behav Brain Res. 2018;336:177-81.

18. Nithianantharajah J, McKechanie AG, Stewart TJ, Johnstone M, Blackwood $\mathrm{DH}, \mathrm{St}$ Clair D, et al. Bridging the translational divide: identical cognitive touchscreen testing in mice and humans carrying mutations in a diseaserelevant homologous gene. Sci Rep. 2015;5:14613.

19. Horner AE, Heath CJ, Hvoslef-Eide M, Kent BA, Kim CH, Nilsson SR, et al. The touchscreen operant platform for testing learning and memory in rats and mice. Nat Protoc. 2013;8:1961-84.

20. Oomen CA, Hvoslef-Eide M, Heath CJ, Mar AC, Horner AE, Bussey TJ, et al. The touchscreen operant platform for testing working memory and pattern separation in rats and mice. Nat Protoc. 2013;8:2006-21.

21. Bussey TJ, Padain TL, Skillings EA, Winters BD, Morton AJ, Saksida LM. The touchscreen cognitive testing method for rodents: how to get the best out of your rat. Learn Memory. 2008;15:516-23.

22. Coba MP, Komiyama NH, Nithianantharajah J, Kopanitsa MV, Indersmitten T, Skene NG, et al. TNiK is required for postsynaptic and nuclear signaling pathways and cognitive function. J Neurosci. 2012;32:13987-99.

23. Kim CH, Heath CJ, Kent BA, Bussey TJ, Saksida LM. The role of the dorsal hippocampus in two versions of the touchscreen automated paired associates learning (PAL) task for mice. Psychopharmacology. 2015:232:3899-910.

24. Romberg C, Horner AE, Bussey TJ, Saksida LM. A touch screen-automated cognitive test battery reveals impaired attention, memory abnormalities, and increased response inhibition in the TgCRND8 mouse model of Alzheimer's disease. Neurobiol Aging. 2013;34:731-44.

25. Mouri A, Ukai M, Uchida M, Hasegawa S, Taniguchi M, Ito T, et al. Juvenile social defeat stress exposure persistently impairs social behaviors and neurogenesis. Neuropharmacology. 2018;133:23-37.

26. Franklin KBJ, Paxinos G. The mouse brain in stereotaxic coordinates, 3rd edn. San Diego: Academic Press; 2007.

27. Mizoguchi H, Katahira K, Inutsuka A, Fukumoto K, Nakamura A, Wang T, et al. Insular neural system controls decision-making in healthy and methamphetamine-treated rats. Proc Natl Acad Sci USA. 2015;112:E3930-9.

28. McTighe SM, Mar AC, Romberg C, Bussey TJ, Saksida LM. A new touchscreen test of pattern separation: effect of hippocampal lesions. NeuroReport. 2009;20:881-5.

29. Talpos JC, Winters BD, Dias R, Saksida LM, Bussey TJ. A novel touchscreenautomated paired-associate learning (PAL) task sensitive to pharmacological manipulation of the hippocampus: a translational rodent model of cognitive impairments in neurodegenerative disease. Psychopharmacology. 2009;205:157-68.

30. Saito T, Saido TC. Neuroinflammation in mouse models of Alzheimer's disease. Clin Exp Neuroimmunol. 2018;9:211-8.

31. Deng W, Mayford M, Gage FH. Selection of distinct populations of dentate granule cells in response to inputs as a mechanism for pattern separation in mice. eLife. 2013;2:e00312.

32. Yassa MA, Stark CE. Pattern separation in the hippocampus. Trends Neurosci. 2011;34:515-25.

33. Duncan K, Ketz N, Inati SJ, Davachi L. Evidence for area CA1 as a match/ mismatch detector: a high-resolution fMRI study of the human hippocampus. Hippocampus. 2012;22:389-98.

34. Terry RD, Masliah E, Salmon DP, Butters N, DeTeresa R, Hill R, et al. Physical basis of cognitive alterations in Alzheimer's disease: synapse loss is the major correlate of cognitive impairment. Ann Neurol. 1991;30:572-80.

35. DeKosky ST, Scheff SW. Synapse loss in frontal cortex biopsies in Alzheimer's disease: correlation with cognitive severity. Ann Neurol. 1990;27:457-64.

36. Kerchner GA, Hess CP, Hammond-Rosenbluth KE, Xu D, Rabinovici GD, Kelley DA, et al. Hippocampal CA1 apical neuropil atrophy in mild Alzheimer disease visualized with 7-T MRI. Neurology. 2010;75:1381-7.

37. Mueller SG, Schuff N, Yaffe K, Madison C, Miller B, Weiner MW. Hippocampal atrophy patterns in mild cognitive impairment and Alzheimer's disease. Hum Brain Mapp. 2010;31:1339-47.

38. Zhu H, Yan H, Tang N, Li X, Pang P, Li H, et al. Impairments of spatial memory in an Alzheimer's disease model via degeneration of hippocampal cholinergic synapses. Nat Commun. 2017:8:1676.

39. Namba T, Ming GL, Song H, Waga C, Enomoto A, Kaibuchi K, et al. NMDA receptor regulates migration of newly generated neurons in the adult hippocampus via Disrupted-In-Schizophrenia 1 (DISC1). J Neurochem. 2011;118:34-44.

40. Kesner RP, Lee I, Gilbert P. A behavioral assessment of hippocampal function based on a subregional analysis. Rev Neurosci. 2004;15:333-51. 
41. Clelland CD, Choi M, Romberg C, Clemenson GD Jr, Fragniere A, Tyers P, et al. A functional role for adult hippocampal neurogenesis in spatial pattern separation. Science. 2009;325:210-3.

42. Sahay A, Scobie KN, Hill AS, O'Carroll CM, Kheirbek MA, Burghardt NS, et al. Increasing adult hippocampal neurogenesis is sufficient to improve pattern separation. Nature. 2011;472:466-70.

43. Ally BA, Hussey EP, Ko PC, Molitor RJ. Pattern separation and pattern completion in Alzheimer's disease: evidence of rapid forgetting in amnestic mild cognitive impairment. Hippocampus. 2013:23:1246-58.

44. Wesnes KA, Annas P, Basun H, Edgar C, Blennow K. Performance on a pattern separation task by Alzheimer's patients shows possible links between disrupted dentate gyrus activity and apolipoprotein E in4 status and cerebrospinal fluid amyloid-beta42 levels. Alzheimer's Res Therapy. 2014;6:20.

45. O'Leary JD, Hoban AE, Cryan JF, O'Leary OF, Nolan YM. Differential effects of adolescent and adult-initiated voluntary exercise on context and cued fear conditioning. Neuropharmacology. 2019;145:49-58.

46. Choi SH, Bylykbashi E, Chatila ZK, Lee SW, Pulli B, Clemenson GD, et al. Combined adult neurogenesis and BDNF mimic exercise effects on cognition in an Alzheimer's mouse model. Science. 2018. https://doi. org/10.1126/science.aan8821.

47. Hueston CM, O'Leary JD, Hoban AE, Kozareva DA, Pawley LC, O'Leary OF, et al. Chronic interleukin-1 beta in the dorsal hippocampus impairs behavioural pattern separation. Brain Behav Immun. 2018;74:252-64.

48. Zhuo JM, Tseng HA, Desai M, Bucklin ME, Mohammed Al, Robinson NT, et al. Young adult born neurons enhance hippocampal dependent performance via influences on bilateral networks. eLife. 2016;5:e22429.

49. Blackwell AD, Sahakian BJ, Vesey R, Semple JM, Robbins TW, Hodges JR. Detecting dementia: novel neuropsychological markers of preclinical Alzheimer's disease. Dement Geriatr Cogn Disord. 2004;17:42-8.
50. Sahakian BJ, Morris RG, Evenden JL, Heald A, Levy R, Philpot M, et al. A comparative study of visuospatial memory and learning in Alzheimertype dementia and Parkinson's disease. Brain J Neurol. 1988;111(Pt 3):695-718

51. Swainson R, Hodges JR, Galton CJ, Semple J, Michael A, Dunn BD, et al. Early detection and differential diagnosis of Alzheimer's disease and depression with neuropsychological tasks. Dement Geriatr Cogn Disord. 2001;12:265-80.

52. Mu Y, Gage FH. Adult hippocampal neurogenesis and its role in Alzheimer's disease. Mol Neurodegener. 2011;6:85.

53. Piiponniemi TO, Bragge T, Vauhkonen EE, Vartiainen P, Puolivali JT, Sweeney PJ, et al. Acquisition and reversal of visual discrimination learning in APPSwDI/Nos2(-/-) (CVN) mice. Neurosci Lett. 2017;650:126-33.

54. Van den Broeck L, Hansquine P, Callaerts-Vegh Z, D'Hooge R. Impaired reversal learning in APPPS1-21 mice in the touchscreen visual discrimination task. Front Behav Neurosci. 2019;13:92.

55. Graybeal C, Feyder M, Schulman E, Saksida LM, Bussey TJ, Brigman JL, et al. Paradoxical reversal learning enhancement by stress or prefrontal cortical damage: rescue with BDNF. Nat Neurosci. 2011;14:1507-9.

56. Chudasama Y, Robbins TW. Dissociable contributions of the orbitofrontal and infralimbic cortex to pavlovian autoshaping and discrimination reversal learning: further evidence for the functional heterogeneity of the rodent frontal cortex. J Neurosci. 2003;23:8771-80.

57. Jones B, Mishkin M. Limbic lesions and the problem of stimulus-reinforcement associations. Exp Neurol. 1972;36:362-77.

\section{Publisher's Note}

Springer Nature remains neutral with regard to jurisdictional claims in published maps and institutional affiliations.
Ready to submit your research? Choose BMC and benefit from:

- fast, convenient online submission

- thorough peer review by experienced researchers in your field

- rapid publication on acceptance

- support for research data, including large and complex data types

- gold Open Access which fosters wider collaboration and increased citations

- maximum visibility for your research: over $100 \mathrm{M}$ website views per year

At BMC, research is always in progress.

Learn more biomedcentral.com/submissions 